\title{
The prevalence of lymphoid follicles in Helicobacter pylori associated gastritis in patients with ulcers and non-ulcer dyspepsia
}

\author{
A M Zaitoun
}

Department of Al Qassimi Hospital, Sharjah. Sharjah, A M Zaitoun

Correspondence to: Dr A M Zaitoun, Mayday Healthcare NHS Trust, Mayday Road Thornton Heath,

Accepted for publication 10 May 1994 Surrey, CR7 7YE.

\begin{abstract}
Aims-To determine the prevalence of lymphoid follicles in Helicobacter pylori positive and negative gastritis in antral and body type gastric mucosa in patients with non-ulcer dyspepsia (NUD), duodenal ulcer, or gastric ulcer; to correlate follicle presence with patient age; to evaluate the correlation between the prevalence of lymphoid follicles and active and inactive gastritis and its severity; and to assess the positive predictive value of lymphoid follicle prevalence with respect to $H$ pylori infection.
\end{abstract}

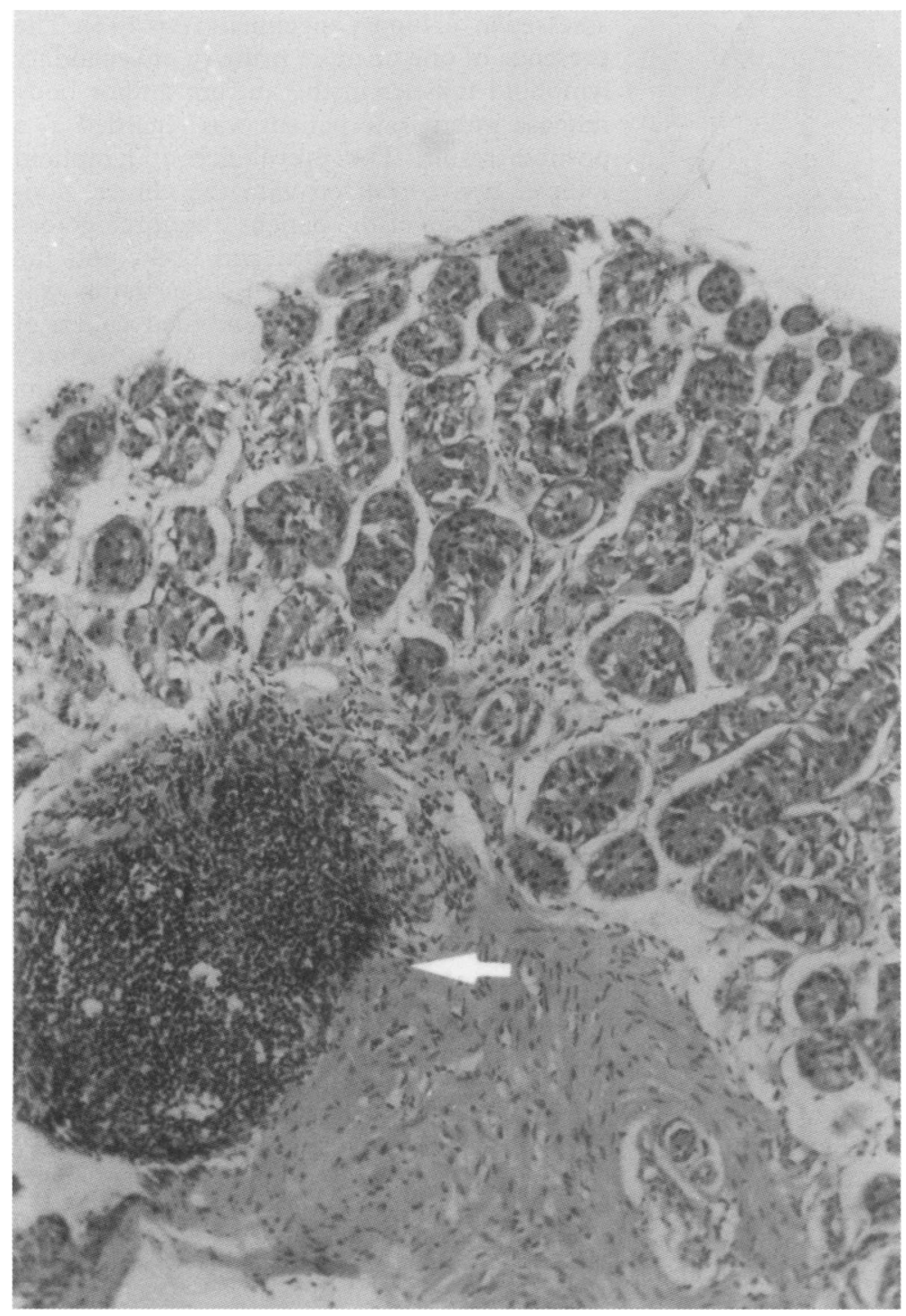

Figure 1 A primary lymphoid follicle in a normal body type gastric biopsy from patient with $H$ pylori associated antral gastritis (haematoxylin and eosin).
Methods-Gastric biopsy specimens, graded according to the Sydney system, from 337 patients were studied.

Results-Lymphoid follicles occurred more often in antral mucosa $(\mathbf{7 8 \%})$ than in body type mucosa (41\%) and were observed in $85 \%$ of patients with $H$ pylori positive gastritis. There was no significant difference between NUD and gastric and duodenal ulcer disease with regard to the presence of lymphoid follicles. The positive predictive value of the presence of lymphoid follicles in $\mathrm{H}$ pylori infection was $96 \%$. Lymphoid follicles were more commonly observed in patients aged between 10 and 29 years. Lymphoid follicles were more frequently found in pangastritis of all subtypes than in antral gastritis and also in active gastritis than in inactive gastritis. The presence of lymphoid follicles correlated strongly with the degree and severity of gastritis.

Conclusion-Lymphoid follicles are a constant morphological feature of $H$ pylori associated gastritis.

( $($ Clin Pathol 1995;48:325-329)

Keywords: Lymphoid follicles, Helicobacter pylori, gastritis.

Chronic gastritis is a histological lesion commonly encountered by the pathologist during routine interpretation of gastric biopsy specimens. Since the discovery in 1983 that $\mathrm{Hel}$ icobacter pylori is a major cause of chronic gastritis, much has been learned about this condition. Many classifications of chronic gastritis are currently in use. The Sydney system for grading gastritis has advantages over other systems as it incorporates aetiology, morphology and topography in a single classification. ${ }^{1}$ In both the classifications of Stolte and Heilman ${ }^{2}$ and in the Sydney system the occurrence of lymphoid follicles in gastric biopsy specimens is regarded as an important, but ungraded, variable which is commonly seen in Helicobacter associated gastritis.

Follicular gastritis is characterised by the presence of reactive lymphoid follicles in inflamed gastric biopsy specimens. The term was first described by Konjetzny et al in 1928 as quoted by Stolte and Eidt. ${ }^{3}$ However, Day ${ }^{4}$ regarded the presence of lymphoid follicles as a descriptive rather than a distinct entity of chronic gastritis. The prevalence of lymphoid follicles has been studied previously. They have 


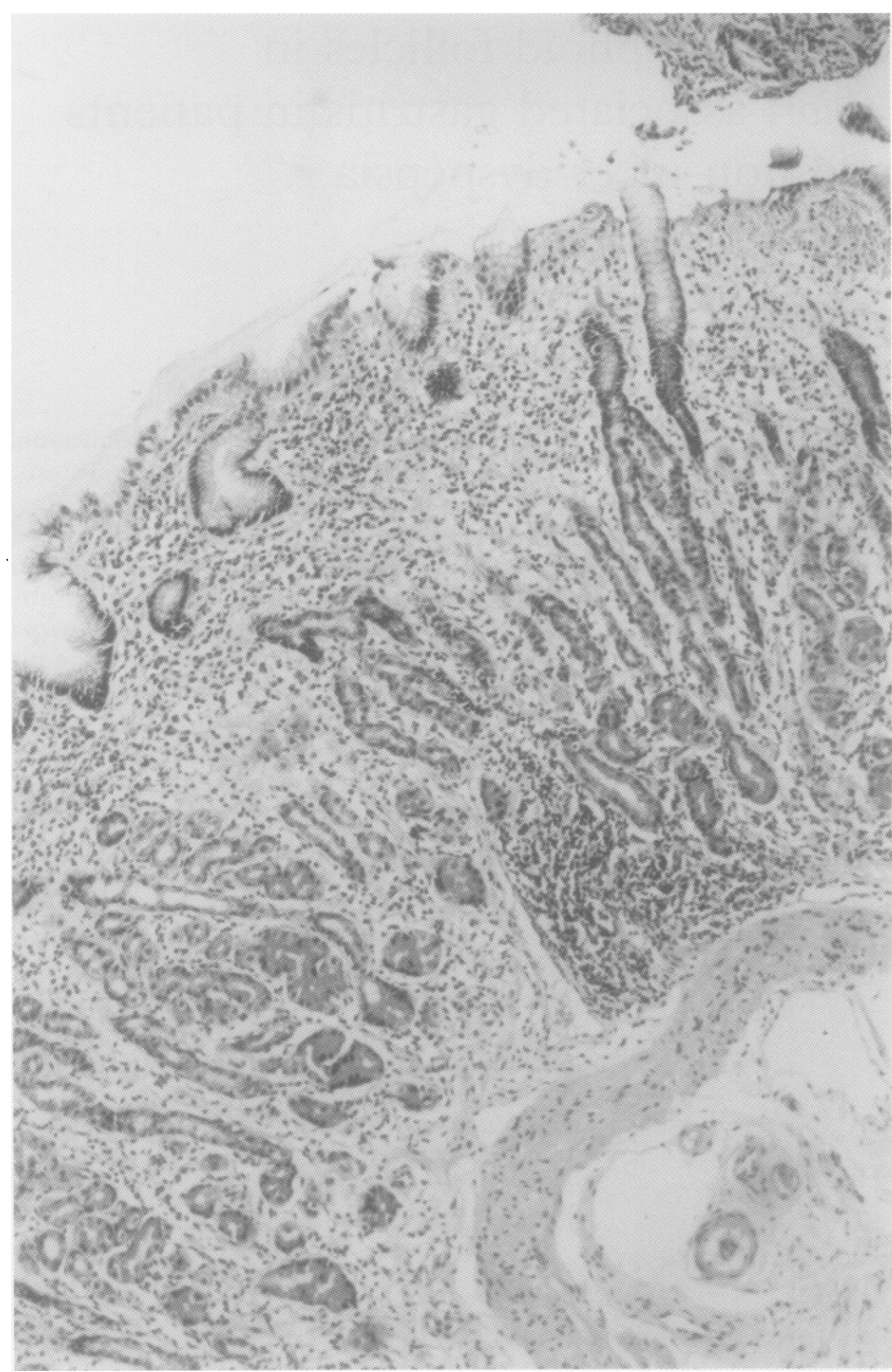

Figure 2 Primary lymphoid follicle in an antral biopsy specimen from a patient with $H$ pylori associated gastritis (haematoxylin and eosin).

been identified in $27.4 \%,{ }^{5} 54 \%^{3}$ and $66 \%^{6}$ of patients with $H$ pylori associated gastritis. However, the occurrence of lymphoid follicles is not restricted to $H$ pylori associated gastritis. ${ }^{5}$ In a more recent study ${ }^{7}$ careful examination of multiple specimens revealed lymphoid follicles in the gastric mucosa of all patients with $H$ pylori infection. The frequency of lymphoid follicles was found to be higher in the antrum than the body in patients with duodenal and gastric ulcer disease. ${ }^{78}$

The aims of this study were to evaluate the occurrence of lymphoid follicles in gastric biopsy specimens from patients with peptic ulcer disease and non-ulcer dyspepsia (NUD); to study the correlation between the formation of lymphoid follicles, the age of the patients, and the activity and severity of gastritis; and to assess the positive predictive value of lymphoid follicles with respect to $H$ pylori infection.

\section{Methods}

Patients with ulcer disease and NUD attending five gastroenterology clinics in the northern region of the United Arab Emirates (UAE) were included in this study. Of the biopsy specimens taken, 337 fulfilled the criteria of the Sydney system for gastritis and were used in this study. ${ }^{9}$ Patients were also grouped according to their clinical condition: NUD, duodenal ulcer, or gastric ulcer. Biopsy specimens were fixed in $10 \%$ formaldehyde and routinely processed. Paraffin wax sections were cut into sections $4 \mu \mathrm{m}$ thick. Each slide contained three to five sections (nine to 15 sections examined). All specimens contained the full thickness of the mucosa and muscularis mucosae. Sections were stained with haematoxylin and eosin, Diff-3 stain $^{10}$ and alcian blue/periodic acidSchiff stain (AB/PAS). Graded variables included the density of $H$ pylori organisms, inflammation, activity, atrophy, and intestinal metaplasia, which were classified as described previously. ${ }^{1}$ Each variable was divided into three grades (grades I, II and III). The topographical patterns of Helicobacter associated gastritis were also assessed. ${ }^{19}$

Primary (lymphoid aggregate) and secondary (with germinal centres) lymphoid follicles were assessed in all biopsy specimens (figs $1-3$ ). The presence of one or more primary or secondary lymphoid follicles in the antrum and/or body mucosa in any one patient was regarded as a positive result. The prevalence of lymphoid follicles was correlated with the clinical condition, the age of the patient, the three grades of activity in the antrum and body, and the grade of severity of gastritis. The latter was obtained from the mean combined values of the grades of $H$ pylori, inflammation and activity in both the antral and body mucosa. Patients with gastric malignancies (lymphoma or carcinoma) were excluded from the study and none of the patients had tuberculosis or Crohn's disease.

\section{STATISTICS}

Differences between the groups were evaluated using the $\chi^{2}$ test with Yates' correction. The results were considered significant if $p<0.05$ (two tailed probability). The positive predictive value for lymphoid follicles in patients with $H$ pylori infection was obtained using the following formula:

Positive predictive value $=\frac{\text { true positive }}{\text { true positive }+ \text { false positive }} \times 100$

where false positive indicates idiopathic gastritis with lymphoid follicles. The correlation between the prevalence of lymphoid follicles and the grade and severity of gastritis was assessed using regression line analysis $(r=0.91$, two tailed probability $\mathrm{p}=0.0019$, intercept $\mathrm{a}=$ $57 \cdot 43$, slope $b=6 \cdot 16$ ).

\section{Results}

In all gastric biopsy specimens examined the percentage of secondary lymphoid follicles was 


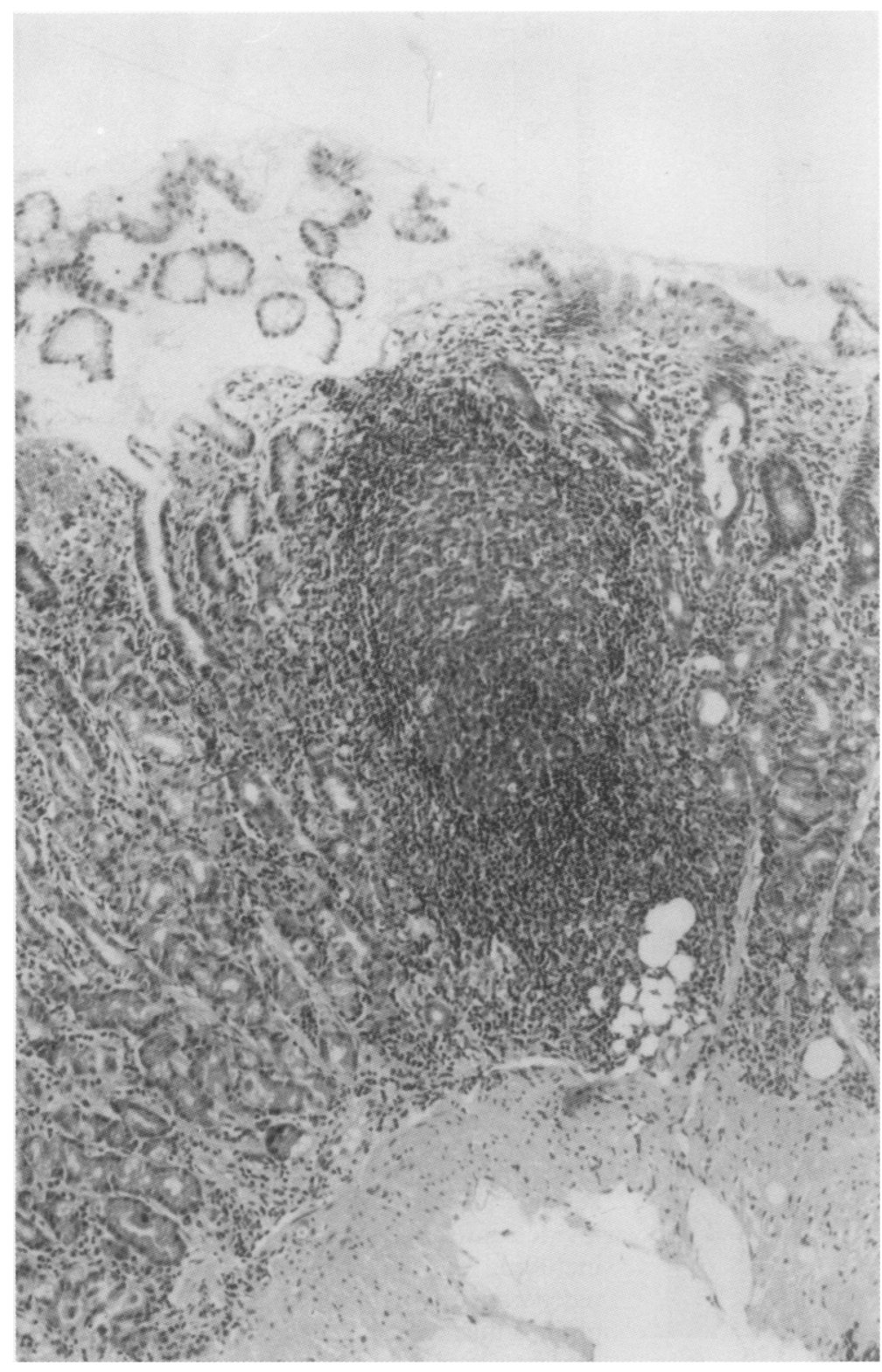

Figure 3 Secondary lymphoid follicle in a biopsy specimen with $H$ pylori associated gastritis (haematoxylin and eosin).

$62 \%$ compared with $38 \%$ for primary lymphoid follicles. In $89 \%$ of biopsy specimens the lymphoid follicles were situated close to the muscularis mucosae. In three patients with antral gastritis lymphoid follicles were observed in corporal biopsy specimens which had no evidence of inflammation (fig 1).

\section{PREVALENCE OF LYMPHOID FOLLICLES WITH} RESPECT TO CLINICAL CONDITION

The overall prevalence of lymphoid follicles in gastric biopsy specimens from 337 patients with peptic ulcer disease and NUD is shown in fig 4. In patients with NUD and $H$ pylori positive

Prevalence of lymphoid follicles (\%) in the antrum and corpus of 294 patients with $H$ pylori associated gastritis with respect to the grade of activity (total number of cases shown in brackets)

\begin{tabular}{|c|c|c|c|c|c|}
\hline \multirow[b]{2}{*}{ Site } & \multirow{2}{*}{$\begin{array}{l}\text { Inactive gastritis } \\
\text { (Grade 0) }\end{array}$} & \multicolumn{3}{|c|}{ Active gastritis } & \multirow{2}{*}{$\begin{array}{l}\text { Active gastritis } \\
\text { (all grades) }\end{array}$} \\
\hline & & Grade I & Grade II & Grade III & \\
\hline $\begin{array}{l}\text { Antrum } \\
\text { Body }\end{array}$ & $\begin{array}{l}22(16 / 73) \\
32(55 / 172)\end{array}$ & $\begin{array}{l}83(99 / 120) \\
52(47 / 90)\end{array}$ & $\begin{array}{l}81(58 / 72) \\
48(12 / 25)\end{array}$ & $\begin{array}{l}86(25 / 29) \\
86(6 / 7)\end{array}$ & $\begin{array}{l}82(182 / 221) \\
53(65 / 122)\end{array}$ \\
\hline
\end{tabular}

gastritis lymphoid follicles were seen in the antrum in 162 of $206(79 \%)$ and in the body in 91 of 206 (44\%). The presence in lymphoid follicles was similarly higher in the antrum than in the body in all other patients with duodenal and gastric ulcer disease. The overall incidence of lymphoid follicles was $87 \%$ in patients with NUD, $82 \%$ in those with duodenal and $83 \%$ in those with gastric ulcer disease. Lymphoid follicles were seen in the antral mucosa in $78 \%$ of patients and in body type mucosa in $41 \%$ ( $p<0.0001)$. The number of cases with $H$ pylori negative gastritis was too small for any conclusions to be drawn with regard to clinical condition but the overall incidence of lymphoid follicles was $47 \%$ compared with $85 \%$ in $H$ pylori positive gastritis $(\mathrm{p}<0.0001)$. Lymphoid follicles were detected in 11 of 24 cases of idiopathic gastritis. The positive predictive value of lymphoid follicles in $H$ pylori infection was $96 \%$.

\section{PATIENT AGE}

Figure 5 shows the age specific prevalence of lymphoid follicles in 294 patients with $H$ pylori associated gastritis. The incidence of lymphoid follicles was highest $(100 \%)$ in those aged between 10 and 19 years and varied between 77 and $89 \%$ in patients aged 20 years and over.

\section{TOPOGRAPHICAL PATTERNS OF GASTRITIS}

The presence of lymphoid follicles with respect to the topographical patterns of $H$ pylori associated gastritis is shown in fig 6 . The incidence of lymphoid follicles decreased from $100 \%$ in those with predominantly corporal pangastritis, to $89 \%$ in predominantly antral pangastritis, to $84 \%$ in pangastritis, and to $66 \%$ in antral gastritis alone (pangastritis $v$ antral gastritis $p<0.03$; predominantly antral pangastritis $v$ antral gastritis $\mathrm{p}<0.001$; predominantly corporal pangastritis $v$ antral gastritis; NS).

\section{ACTIVE AND INACTIVE GASTRITIS}

The prevalence of lymphoid follicles in the antrum and body in active and inactive gastritis is presented in the table. In the antrum lymphoid follicles were present in $22 \%(16 / 73)$ of patients with inactive gastritis and in $82 \%$ of those with active gastritis (all grades) $(p<0.0006)$. In the body the incidence of lymphoid follicles increased from 32\% (55/ 172 ) in patients with inactive gastritis to $53 \%$ in those with active gastritis $(p<0.0003)$. The differences in the prevalence of lymphoid follicles between antral and body mucosa with regard to inactive and active gastritis were also statistically significant $(p<0.0001)$.

\section{DEGREE OF GASTRITIS}

The incidence of lymphoid follicles in relation to the degree of gastritis in both antrum and body is summarised in fig 7 . The presence of lymphoid follicles in $H$ pylori associated gastritis correlated significantly with the degree of gast- 


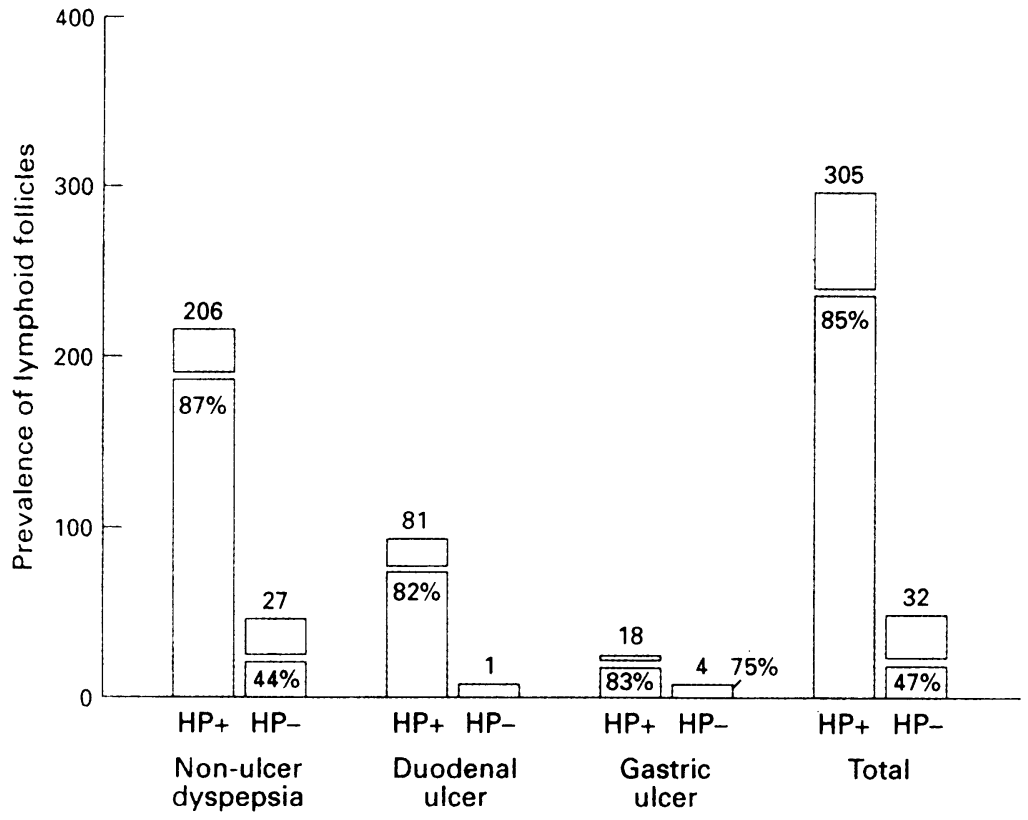

Figure 4 Prevalence of lymphoid follicles in 377 patients with peptic ulcer disease and NUD. $H P=H$ pylori

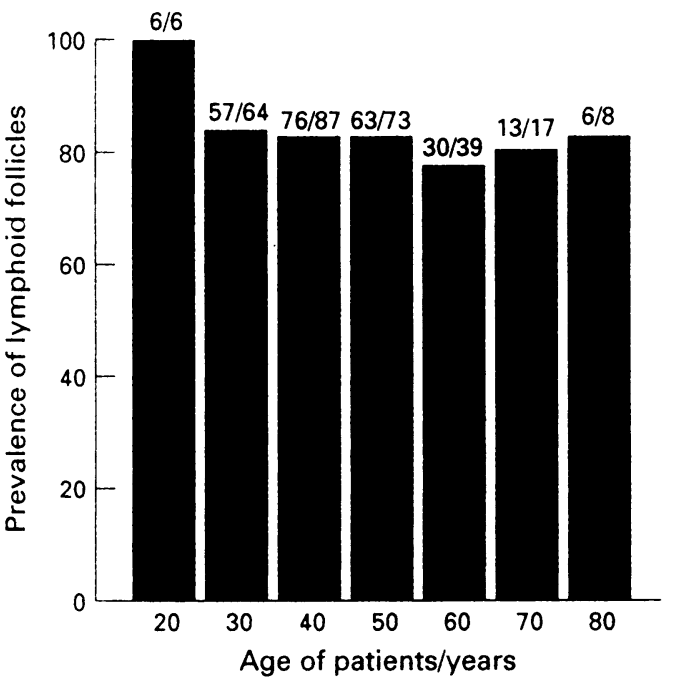

Figure 5 Lymphoid follicles in H pylori associated gastritis with respect to age.

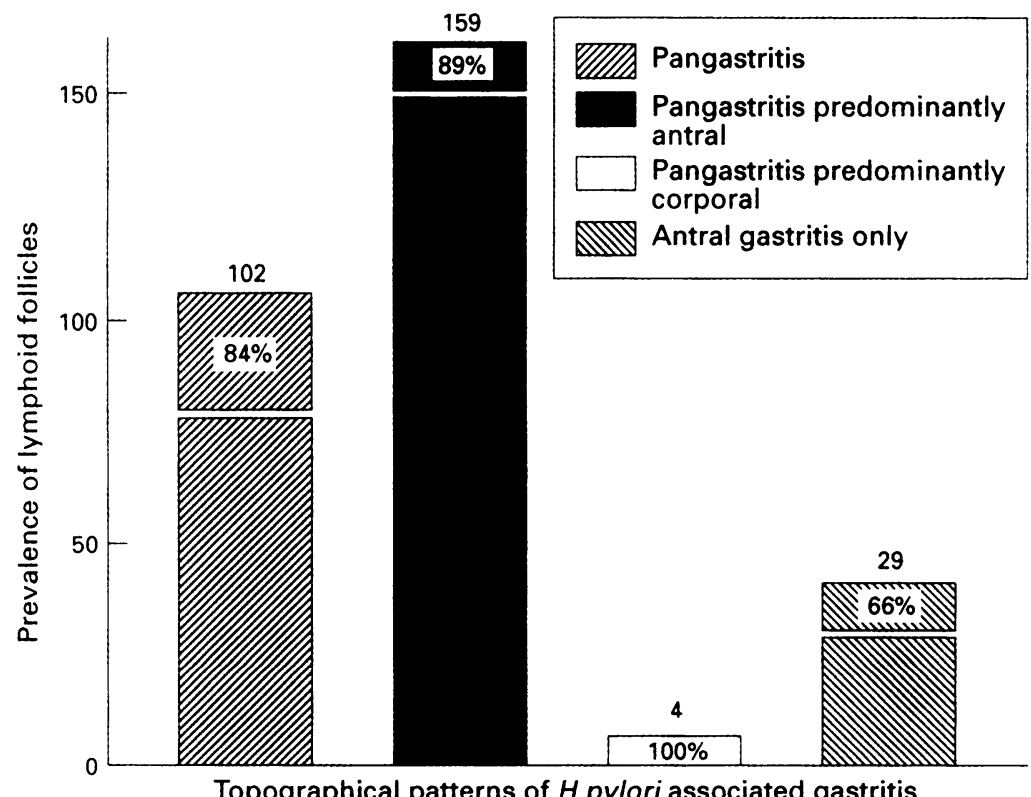

Figure 6 The prevalence of lymphoid follicles in H pylori associated gastritis with respect to the topographical patterns.

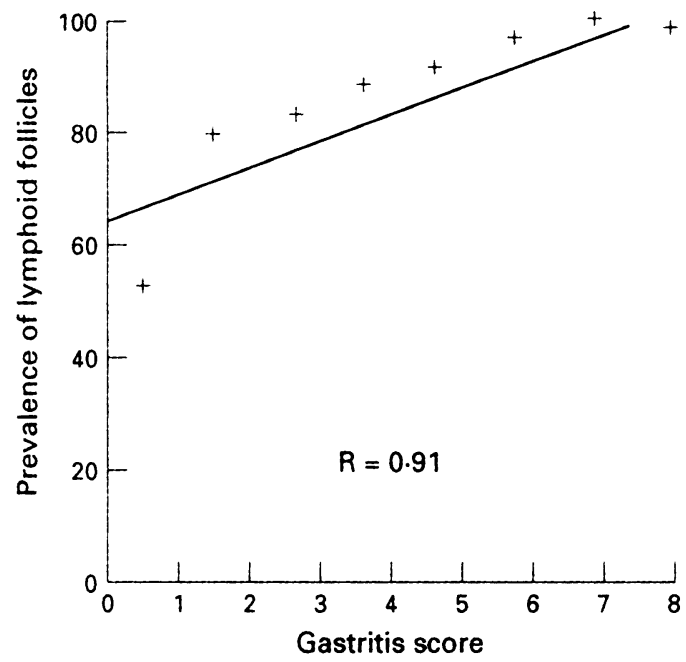

Figure 7 Correlation between the prevalence of lymphoid follicles and the grade and severity of gastritis in 294 patients with $H$ pylori associated gastritis.

ritis. The incidence increased in an exponential manner from $50 \%$ for grade I gastritis to $89 \%$ for grade IV to $100 \%$ for grades VII and VIII $(\mathrm{p}=0.0019)$.

\section{Discussion}

The morphological appearance of chronic gastritis is well known and Helicobacter associated gastritis represents the most common form of this condition. ${ }^{1}$

In the Sydney system for classifying chronic gastritis the density of $H$ pylori, inflammation, activity, atrophy, and intestinal metaplasia are divided into three grades. Ungraded variables including the presence of lymphoid follicles, regenerative hyperplasia, ulceration, and eosinophilic infiltration are commonly seen in Helicobacter associated gastritis."

This study has demonstrated the presence of lymphoid follicles in $85 \%$ of patients with $H$ pylori positive gastritis; this figure is $47 \%$ in patients with $H$ pylori negative gastritis $(p<0 \cdot 0001)$. These figures are higher than those reported by other authors ${ }^{356}$ who recorded the presence of lymphoid follicles in gastric biopsy specimens in $27 \cdot 4 \%,{ }^{5} 54 \%{ }^{3}$ and $66 \%{ }^{6}$ of patients with Helicobacter associated gastritis. In two of those studies ${ }^{36}$ the presence of lymphoid follicles was evaluated in the antrum only. In the current study at least four gastric biopsy specimens from the antrum and body were evaluated, offering increased scope for detection.

No significant difference in the presence of lymphoid follicles were noted in biopsy material from patients with NUD $(87 \%)$, and duodenal $(82 \%)$ or gastric $(83 \%)$ ulcer disease and $H$ pylori associated gastritis. Lymphoid follicles were more common in the antrum $(78 \%)$ than in the body mucosa $(41 \%)$ in all cases studied ( $p>0.0001)$. Lymphoid follicles were also identified in $H$ pylori negative gastritis in $47 \%$ of cases. However, the number of cases with $H$ pylori negative gastritis was small and in some this condition may have arisen because of a previous infection with $H$ pylori, which cannot 
be evaluated by histological methods alone. ${ }^{12}$ The positive predictive value of lymphoid follicles in $H$ pylori infection was $96 \%$. This is in accord with a recent study by Genta $e t a^{7}$ who found that lymphoid follicles were present in gastric biopsy specimens from all patients infected with $H$ pylori, detected using the mapped gastric biopsy specimen technique.

The results of this study confirm the findings of two recent studies ${ }^{78}$ which showed that the prevalence of lymphoid follicles was higher in the antral mucosa than the body mucosa in patients with duodenal and gastric ulcer disease. In addition, the current study demonstrated similar findings in patients with NUD. A possible explanation for the lower prevalence of lymphoid follicles in the body mucosa may be related to the degree of gastric inflammation which is higher in the antrum than the body. ${ }^{9}$ The presence of lymphoid follicles in all cases of predominantly corporal pangastritis (fig 6) supports such a hypothesis.

The formation of lymphoid follicles in $\mathrm{H}$ pylori associated gastritis occurred in $100 \%$ of patients aged between 10 and 19 years. Mahony and Littlewood ${ }^{13}$ reported that lymphoid follicles are a prominent feature of biopsy specimens from paediatric patients with $H$ pylori associated antral gastritis and correlate well with the presence of antral nodularity on endoscopy. In another study follicular gastritis was seen in 10 of 11 paediatric patients with antral nodularity ${ }^{14}$ giving a sensitivity of $96.4 \%$ for the diagnosis of $H$ pylori infection in the paediatric age group. ${ }^{15}$ The incidence of lymphoid follicles decreased to $89 \%$ in patients aged between 20 and 29 years and to approximately $82 \%$ thereafter.

Another important finding of this study is the significant correlation between the topographical pattern of $H$ pylori associated gastritis and the presence of lymphoid follicles. Predominantly antral pangastritis was associated with lymphoid follicles in $89 \%$ of specimens compared with $66 \%$ for antral gastritis alone $(p<0.0008)$.

A significant difference in the presence of lymphoid follicles between inactive and active gastritis was noted in both the antrum (22v $82 \% ; \mathrm{p}<0.0006)$ and body (32 v 53\%; $\mathrm{p}<0.0003)$. These results are in accord with those reported previously. ${ }^{36}$

The data suggest that the relation between the presence of lymphoid follicles and the degree of gastritis is linear $(r=0.91)$. The higher degree of gastritis, the higher the incidence of lymphoid follicles. These results are also in keeping with those reported by other authors. ${ }^{6}$

Some authors ${ }^{51617}$ have reported that many of the lymphoid follicles are present deep within the gastric mucosa, as was the case in this study where $89 \%$ of the lymphoid follicles (primary and secondary) occurred very close to the base of gastric mucosa. Thus, most lymphoid foll- icles, if not all, originate in the lower part of the mucosa close to the muscularis mucosae. This may be explained by the presence of a lymphatic vessel plexus in the deeper part of the mucosa. Similar findings have been observed in experimental animals. ${ }^{16}$ Radin $e t a l{ }^{16}$ found that lymphoid follicle formation was most prominent in the antrum and close to basal border of the lamina propria.

In conclusion, lymphoid follicles are a constant feature of $H$ pylori infection. There was no difference in the prevalence of lymphoid follicles in patients with NUD, duodenal ulcer or gastric ulcer disease. The positive predictive value of lymphoid follicles in $H$ pylori infection was $96 \%$. Lymphoid follicles were detected more frequently in the antrum than in the body. They were also more frequent in the second decade of life and in active gastritis more than in inactive gastritis. The presence of lymphoid follicles was strongly correlated with the degree and severity of gastritis. Lymphoid follicles were present in uninflamed body mucosa from patients with $H$ pylori associated antral gastritis.

1 Price AB. The Sydney System: Histological division. $f$ Gastroenterol Hepatol 1991;6:209-22.

2 Stolte M, Heilmann KL. New classification of gastritis. Leber Magen Darm 1989;19:220-6.

3 Stolte M, Eidt S. Lymphoid follicles in antral mucosa: immune response to Campylobacter pylori. f Clin Pathol 1989;42:1269-71.

4 Day DW. The stomach. In: Morson BC, ed. Alimentary tract. Volume 3. Systemic pathology. Edinburgh: Churchil Livingstone, 1987:149-228.

5 Wyatt JI, Rathbone BJ. Immune response of the gastric mucosa to Campylobacter pylori. Scand $\mathcal{f}$ Gastroenterol 1988;23(Suppl 142):44-9.

6 Hauke C, Grabner W, Grosse M, Stolte M. Lymph follicle formation and development of intestinal metaplasia in antrum mucosa as a reaction to Helicobacter pylori infection [in German]. Leber Magen Darm 1991;21:156-60.

7 Genta RM, Hamner HW, Graham DY. Gastric lymphoid follicles in Helicobacter pylori infection: Frequency, distribution and response to triple therapy. Hum Pathol 1993; 24:577-83.

8 Eidt $S$, Stolte $M$. Prevalence of lymphoid follicles and aggregates in Helicobacter pylori gastritis in antral and body mucosa. $f$ Clin Pathol 1993;46:832-5.

9 Zaitoun AM. Histological study of chronic gastritis from the United Arab Emirates using the Sydney system of the United Arab Emirates using the Sydrey

10 Zaitoun AM. Use of Romanowsky type (Diff-3) stain for detecting Helicobacter pylori in smears and tissue sections. f Clin Pathol 1992;45:448-9.

11 Dixon MF. Helicobacter pylori and chronic gastritis. In: Rathbone BJ, Heatley RV, eds. Helicobacter pylori and gastroduodenal disease. 2nd edn. Oxford: Blackwell Scientific Publications, 1992:124-39.

12 Karnes WEJ, Samlof IM, Siurala M, Kekki M, Sipponen P, Kim SWR, et al. Positive serum antibody and negative tissue staining for Helicobacter pylori in subjects with tissue staining for Helicobacter pylori in subjects with

13 Mahony MJ, Littlewood JM. Helicobacter pylori in a paediatric population. In: Rathbone BJ, Heatley RV, eds. Helicobacter pylori and gastroduodenal disease. 2nd edn.

14 De Giacomo C, Fiocca R, Villani L, Lisato L, Licardi G, Diegoli N, et al. Helicobacter pylori infection and chronic gastritis. Clinical, serological and histological correlations in children treated with amoxicillin and colloidal bismuth substrate. F Pediatr Gastroenterol Nutr 1990;11:310-16.

15 Conti-Nibali S, Sferlazzas C, Fera MT, Saitta G, Tedesch A, Magazzu G. Helicobacter pylori infection: a simplified A, Magazz 16 Radin MJ, Eaton KA, Krakowka S, Morgan DR, Lee A, Otto G, et al. Helicobacter pylori gastritis infection in Otto G, et al. Helicobacter pylori gastritis infection in

17 Wotherspoon AC, Ortiz-Hidalgo C, Falzon MR, Isaacson PT. Helicobacter pylori-associated gastritis and primary B-cell gastric lymphoma. Lancet 1991;338:1175-6. 